\title{
DE LA GRAMÁTICA ORACIONAL A LA GRAMÁTICA DISCURSIVA EN EL AULA DE ESPAÑOL COMO LENGUA EXTRANJERA
}

\author{
Patricia Guillén Solano
}

\begin{abstract}
RESUMEN
El artículo aborda los principios y características de la gramática discursiva, con el fin de analizar la necesidad de incorporar este tipo de gramática en el aula de español como lengua extranjera e ir más allá de la perspectiva oracional. Además, se estudian diferentes aspectos relacionados con el enfoque discursivo, tales como contexto y deixis. Finalmente, para ejemplificar la aplicación de dicho enfoque, se analiza el uso de los pronombres personales de sujeto a partir de una conversación espontánea entre dos hablantes nativos del español.

Palabras clave: gramática discursiva, gramática oracional, deixis, contexto,conversación.
\end{abstract}

\begin{abstract}
This article describes the characteristics and principles of the discourse grammar in order to analyze the necessity to incorporate this grammar into the class of Spanish as a foreign language and to go beyond the sentence grammar perspective. Moreover, different components related to the discourse approach, such as deixis and context, are studied. Finally, in order to exemplify the application of the discourse grammar principles, the use of subject personal pronouns in a spontaneous conversation between Spanish native speakers is analyzed.

Key words: discourse grammar, sentence grammar, context, deixis, conversation.
\end{abstract}

\section{Introducción}

Tradicionalmente, el término gramática ha sido definido de forma muy limitada en la clase de español como lengua extranjera ${ }^{1}$. Así, la gramática consiste, básicamente, en un conjunto de reglas aisladas que deben memorizarse. Esta cosificación del término se debe, fundamentalmente, al peso del enfoque tradicional en la metodología de lenguas extranjeras.

ML. Patricia Guillén Solano. Profesora del Departamento de Lingüística. Universidad de Costa Rica. Correo electrónico: patrihauster@gmail.com

Recepción: 10- 4- 2010

Aceptación: 15- 5- 2010 
Dentro de este enfoque, la oración es la unidad básica de enseñanza y de práctica de cualquier lengua que quiera aprenderse ${ }^{2}$. En este sentido, no debemos dejar de lado que el enfoque tradicional parte de nociones tanto teóricas como metodológicas que se relacionaron en principio con la enseñanza de lenguas clásicas, fundamentalmente del latín, las cuales se aplicaron a la enseñanza de lenguas extranjeras en general, sobre todo durante el siglo XIX e inicios del siglo XX (Sánchez Pérez 1997: 36). De acuerdo con estas nociones, la gramática permite formalizar el objeto de enseñanza, en tanto lo sistematiza y da cuenta de los contenidos que deben enseñarse. Estos contenidos se presentan y practican mediante el análisis y la construcción de oraciones.

El trabajar a nivel oracional conduce a que se ponga atención especial a la precisión en el uso normativo de la gramática: se explica en detalle una regla gramatical, se muestra una serie de oraciones que ilustran su uso y, finalmente, los estudiantes realizan un ejercicio de aplicación de dicha regla, siguiendo el patrón oracional dado por el profesor. Precisamente, el visualizar la lengua como un conjunto de reglas perfectas es lo que, de acuerdo con Sánchez (1997: 134), le asigna un carácter casi sagrado a los textos y manuales de instrucción: la lengua es tal y como se describe y explica en el libro de texto, a partir de ejemplos normativos.

Claro está que la gramática de una lengua es indispensable como instrumento de enseñanza, sin embargo, durante años el problema ha radicado en que esta se aborde desde una perspectiva de análisis y razonamiento sobre la lengua en sí y no sobre su uso en situaciones comunicativas concretas.

Aun cuando desde el siglo XVI encontramos intentos de contextualizar y oralizar la gramática de una lengua extranjera, recurriendo para ello a la creación de diálogos, tal es el caso de obras como la de Minsheu, de 1599, Pleasant and delightful dialogues in Spanish and English (citado en Sánchez Pérez 1997: 52), es gracias al método audiolingüe cuando, a mediados del siglo XX, el desarrollar en el hablante no nativo la capacidad de expresarse oralmente de forma apropiada se convierte en el principal objetivo dentro de los programas de enseñanza de lenguas extranjeras (Shrum y Glisan 1994: 140).

Dicho método cuestionó también la importancia de la enseñanza de la gramática explícita en el aula, la cual había defendido y puesto en práctica el método de gramática traducción, y se enfocó en el desarrollo de la expresión oral con base en un aprendizaje de estímulo-respuesta: repetición y memorización de diálogos. Sin embargo, los estudiantes no eran capaces de transferir el material memorizado a la comunicación espontánea. El acercamiento cognitivo, en los años sesenta, promovió un uso más significativo del lenguaje y de la creatividad. Esta metodología se basó, en gran parte, en la distinción propuesta por Chomsky entre competencia, el conocimiento intuitivo de reglas de gramática y de cómo opera el sistema lingüístico de una lengua, y la actuación, la habilidad del individuo de producir lenguaje. De acuerdo con esta perspectiva, los estudiantes debían entender las reglas de la lengua antes de poder interactuar y usarla en situaciones específicas. Aun cuando el método cognitivo procuraba la práctica creativa con el lenguaje, las explicaciones y discusiones acerca de las reglas gramaticales y la práctica mecánica dejaban, tal como ocurría con el método de gramática traducción, poco tiempo al desarrollo de la parte comunicativa.

A partir de los años setenta, un planteamiento basado en las necesidades de los estudiantes y en la naturaleza de la comunicación dio paso a un enfoque comunicativo de la enseñanza. Este profundizó la definición de competencia de Chomsky y originó modelos específicos como el de Canale y Swain (citado en Celce-Murcia 2000: 16), que subdivide la 
competencia comunicativa en cuatro áreas: la competencia gramatical, el uso de la gramática apropiada, el vocabulario y la pronunciación; la competencia sociolingüística, el uso de elementos como el estilo, el registro y la entonación, de acuerdo con diferentes contextos; la competencia estratégica, el uso de estrategias comunicativas verbales y no verbales, como los gestos y el circunloquio, para compensar la falta de lenguaje y la competencia discursiva, la habilidad de combinar elementos de la lengua y mostrar cohesión y coherencia dentro de una amplia gama de discursos. El plantear la noción de competencia discursiva facilitó el paso de un estudio de la lengua basado en la oración a uno que asume que es en el discurso donde la lengua se manifiesta como ente social.

En este artículo, se plantea, precisamente, la importancia de incorporar en el aula de ELE las propuestas de la gramática discursiva. Para ello, se recurre a una sistematización de sus características y al análisis, partiendo también desde sus planteamientos, del uso de los pronombres personales de sujeto en una conversación espontánea entre dos hablantes nativos del español.

\section{La gramática discursiva frente a la gramática oracional}

\subsection{Hacia una definición del término gramática}

Gramática es un término con varias acepciones, que difieren tanto en su extensión como en la naturaleza del objeto al que hace referencia [...] Por un lado, hace referencia a un determinado aspecto del lenguaje o de una lengua en particular y, por otro, también designa a la disciplina lingüística que tiene como objeto la descripción y análisis de ese aspecto. (Alcaraz Varó, Martínez Linares 2004: 318)

Rescatamos de esta definición el hecho de que la relación entre lengua y gramática se determina de acuerdo con el objeto al que hace referencia la gramática por sí misma. A este respecto, Crystal (1997: 88) identifica dos posiciones diferentes:

a) En un sentido específico, la gramática es un componente más de la estructura de la lengua, junto con la fonología y la semántica. Vale mencionar que esta es la concepción más tradicional.

b) En un sentido general, popularizado por Chomsky, la gramática incluye todos los aspectos de la estructura de una lengua, dentro de ellos la fonología y la semántica, e introduce el término 'sintaxis' como noción más específica ligada a la gramática.

Otros modelos introducen nuevos componentes, pero siempre a partir de estas dos posiciones, tal es el caso de Halliday (1961), quien plantea tres componentes principales: sustancia, forma y contexto. En su propuesta, es mediante la fonología que la sustancia y la forma se relacionan, al tiempo que la forma se divide en gramática y lexis: la sustancia se plasma en la fonética y el contexto refiere a la semántica.

Crystal (1997), por su parte, sitúa la gramática como un componente más de la lengua, constituida, a su vez, por la morfología y la sintaxis. Cabe destacar que en su modelo, Crystal incorpora la dimensión de la lengua en uso, la cual se relaciona con la estructura de la lengua a través de la pragmática.

Cualquiera que sea la posición que adoptemos, lo cierto es que el término gramática es fundamental a la hora de determinar la estructura de una lengua, e implica, siguiendo a 
Crystal (1997), identificar las unidades con las cuales trabaja y analizar los patrones en los cuales entran estas unidades y las relaciones de significado que conllevan.

\subsection{Más allá de una noción tradicional de gramática: aportes de la gramática funcional a la perspectiva discursiva}

De acuerdo con Serrano (2002: 8), el paradigma formal tradicional concibe la lengua como un conjunto de oraciones, lo cual conlleva, necesariamente, a que en los textos dedicados a la enseñanza del español como lengua extranjera se privilegie el nivel oracional sobre el discursivo. Si partimos de esta premisa, parece claro que se privilegie también el conocimiento de las reglas gramaticales y no el uso discursivo de ellas. Esto produce que la gramática siga viéndose desde un punto de vista ideal, en tanto se considera invariable y constante. Comúnmente, no se abordan casos de modificaciones en las reglas por considerarse incorrectos y arbitrarios. Resulta, por ello, interesante y necesario que en las clases de español como lengua extranjera se contemple que estas variaciones están lejos de la arbitrariedad y resultan, más bien, motivadas.

Los primeros planteamientos de una gramática textual o discursiva datan de las décadas de los sesenta y setenta del siglo XX (Calsamiglia y Tusón 1999: 217) e intentaron extender el análisis gramatical más allá de los límites oracionales. La oración había sido el eje de análisis dentro de las perspectivas estructuralista y generativista. El pasar a un nivel superior del oracional conllevó a que se tuvieran que ampliar los parámetros de análisis que se habían utilizado hasta entonces en la descripción gramatical, incorporando principios y metodología de disciplinas como la pragmática y la sociolinguiística. Las autoras apuntan también que actualmente hay unanimidad en considerar el texto como unidad comunicativa de orden distinto al oracional, pues constituye una unidad semántico-pragmática de sentido, y no solo de significado; una unidad intencional y de interacción, no así el objeto autónomo que había constituido, para teorías anteriores, la oración.

El discurso es el lugar donde la gramática se pone en uso, así como la fuente de donde se forma o "surge" la gramática, a partir de los patrones recurrentes en el discurso (Cumming y Ono 2000: 171). Para abordar el estudio del discurso en la clase de ELE, es necesario entonces partir de una unidad de análisis distinta a la de oración, en su sentido más tradicional. Esta unidad es el enunciado, entendido como el producto concreto y tangible de un proceso de enunciación realizado por un enunciador y destinado a un enunciatario. Este enunciado puede tener o no la forma de lo que tradicionalmente hemos llamado oración: unidad conformada por un verbo y que contiene dos unidades significativas entre las cuales se establece la relación predicativa: el sujeto y el predicado (Alarcos Llorach 1999: 256). El plantear el análisis de enunciados y no de oraciones en su sentido más tradicional, nos permite identificar y explicar muchas más estrategias a nivel discursivo.

Sin duda, existe una clara relación entre el estudio de la gramática a nivel discursivo y las premisas de la gramática funcional. Givon (1995 Vol. I: 2), por ejemplo, plantea que la gramática no es un conjunto de reglas rígidas que deben seguirse para construir oraciones, y la define más bien como un conjunto de estrategias que se emplean para producir comunicación coherente. Quizás la premisa de la gramática funcional que motivó más cambios en la metodología de lenguas extranjeras es la que plantea que el lenguaje es un instrumento de interacción social y, por lo tanto, el estudio del sistema lingüístico no puede separarse del 
contexto de uso. Para Halliday (1973: 7), el enfoque funcional persigue cuáles son los propósitos para los que usamos el lenguaje y cómo somos capaces de lograr estos propósitos a través del habla, la escucha, la lectura y la escritura. El aporte fundamental de esta perspectiva radica en la posibilidad de abordar los fenómenos gramaticales desde un punto de vista realmente comunicativo, lo cual implica encontrar los patrones de modificación y creación de nuevas estrategias sintácticas para luego explicarlos.

En el discurso, se manifiestan elementos que solo desde una perspectiva funcional se pueden analizar, pues la mera descripción formal y normativa resultaría insuficiente. El carácter dinámico del discurso, producto de una actividad social cambiante, da cuenta de la necesidad de incorporar la gramática discursiva en el aula de ELE.

Serrano (2000: 14) afirma que la ausencia de una gramática discursiva del español radica en la importancia que se le ha dado al nivel oracional en tanto este facilita la imposición de regularidades que a nivel discursivo pueden darse o no, negando que la variación es inherente al sistema y tildándola de arbitraria y caprichosa, según los criterios más tradicionales. Sin embargo, la variación presenta tendencias y regularidades que serían objeto de análisis, precisamente, de la gramática del discurso.

\subsection{Principios de la gramática del discurso}

Serrano (2002: 18) esboza las siguientes características de la gramática del discurso:

\subsubsection{Principio de regularidad}

Parte de que existen tendencias que explicitan los mecanismos de la gramática para que determinadas formas adquieran nuevas funciones. Así, "para que una forma adquiera cierta función se tienen que dar una serie de condiciones, bien derivadas de su propia forma, del contexto o de la gramaticalización"3. Para la gramática discursiva, estas condiciones pueden ser sistematizadas, en tanto se presentan de forma regular.

\subsubsection{Principio de funcionalidad}

Todos los estudios a nivel discursivo incorporan tanto la estructura como la función (Schiffrin 1994: 339). De acuerdo con un enfoque funcional, el lenguaje tiene funciones que son externas al sistema lingüístico y, precisamente, estas condicionan la organización interna del sistema en sí. Según el principio de funcionalidad, el análisis gramatical consiste en identificar funciones, explicarlas y relacionarlas con una estructura formal y, por último, establecer las tendencias y las regularidades de esas funciones.

\subsubsection{Principio metodológico}

La metodología parte de una base estrictamente sintáctica, e incorpora, necesariamente, aspectos discursivos y pragmáticos. A este respecto, rescatamos el objetivo central de la pragmática de acuerdo con Escandel Vidal (1996: 13): el estudio de los principios que regulan el uso del lenguaje en la comunicación. Siguiendo esta línea, la sintaxis, necesariamente, se liga al contexto, entendido como el conjunto de factores que organizan, durante el acto comunicativo, los signos lingüísticos según las necesidades puntuales de la comunicación (Vigara Tauste 1996: 17). 
Otro aspecto importante de la metodología de la gramática discursiva es el hecho de que abandona la idea de que cada categoría gramatical está correlacionada con una determinada función, pues seguir esta premisa implicaría dejar de lado la posibilidad de que una misma categoría pueda adoptar diferentes funciones a nivel discursivo. Una gramática del discurso plantea, según Serrano (2002: 33) que se debe tanto determinar categorías gramaticales a nivel formal, como regularizar las funciones comunicativas que estas categorías cumplen discursivamente. A este respecto, la metodología utilizada da cuenta de las regularidades y patrones de interpretación del discurso.

\section{Aplicaciones de la gramática discursiva en la clase de ELE}

El convertir la clase de ELE en una práctica discursiva conlleva mucho más que incorporar terminología diferente, pues involucra la utilización de material didáctico que la ejemplifique y conduzca luego a su uso adecuado. Dentro de todo texto, se establece una serie de relaciones que finalmente terminan por asignarle un significado específico que, a su vez, contiene una función social. Para Brown y Yule (1993: 19), el análisis del discurso es, por necesidad, análisis de la lengua en uso, por lo cual no puede limitarse a la descripción de formas lingüísticas sin tomar en cuenta sus propósitos y funciones. Este tipo de acercamiento no se centra entonces en la determinación de propiedades formales de una lengua, sino en los motivos por los cuáles se utilizan.

La descripción de las formas lingüísticas que expresan contenidos específicos es denominada función descriptiva del lenguaje y se relaciona primariamente con la transmisión de información. Por otro lado, el uso del lenguaje que se relaciona con la expresión de relaciones sociales y actitudes personales pertenece a la función interactiva ${ }^{4}$.

En el caso de la enseñanza de ELE, la tendencia, aun con el advenimiento de enfoques de índole comunicativo, ha sido centrarse en la descripción de las características formales de la lengua. Así, lo común es que en los libros de texto abunden ejemplos construidos por los autores, muy a propósito para que ilustren una característica en particular de la lengua en estudio.

En una perspectiva interactiva, partimos de que los datos provienen de la producción lingüística en contexto, no de la creada por el especialista de la lengua, por lo cual no suele corresponder a oraciones aisladas y perfectas que ejemplifican determinadas reglas gramaticales.

Alejarnos de la manifestación más completa del uso de la gramática, el discurso, hace que la lengua se analice de forma segmentada, aislando cada parte sin ver nunca el conjunto. Mucho se habla de los enfoques comunicativos de la enseñanza de ELE, pero parecemos estar lejos aún de propiciar un contacto con la lengua siempre contextualizado y que parta del estudio de estrategias discursivas específicas.

Siguiendo un enfoque funcionalista discursivo, la gramática se aborda en relación con dos funciones: su función cognitiva, que se relaciona con el manejo de la información, con cómo y por qué se codifica y decodifica de una manera específica; y su función social, que explica cómo se construyen las relaciones sociales dentro de las lenguas, mediante cuáles estrategias morfosintácticas el hablante expresa su mensaje de acuerdo con factores propios del contexto y la situación comunicativa (Sánchez 2006: 262).

La importancia de la incorporación de la gramática discursiva en aula de ELE está relacionada con tratar de ser consecuentes con la propuesta de enfatizar el desarrollo de habilidades comunicativas de acuerdo con distintos tipos de discurso. 


\subsection{Intentos de incorporar el análisis discursivo en el aula de ELE}

Dentro de la enseñanza del español como lengua extranjera, destacan los parámetros establecidos por el Instituto Cervantes en su plan curricular, pues en el apartado 7 rescatan los géneros discursivos para el ámbito específico de la didáctica de lenguas. De acuerdo con su propuesta, los textos se trabajan en el aula tanto desde un enfoque global como en sus aspectos formales más concretos e individualizados (Instituto Cervantes 2006: 305). Plantea también que el hecho de que todas las culturas dispongan de géneros discursivos para comunicarse permite partir de un concepto común-universal que puede manifestarse de formas muy diversas en función del contexto en que se emplee.

En este plan curricular, planteado de forma general para la didáctica del español, se elabora un inventario de géneros orales y escritos con el fin de que el profesor aborde en el aula sus características, su estructura y función, pues "el tratamiento de los textos desde el punto de vista del género al que pertenecen facilita que el alumno perciba de forma global, y en sus distintos componentes, la función comunicativa que cumplen en el contexto social para el que fueron creados" (Instituto Cervantes 2006: 308). El trabajar bajo esta perspectiva permite partir de lo general a lo particular y viceversa, dentro de contextos específicos y tomando en cuenta que los componentes de la lengua actúan siempre de forma simultánea y relacionada.

El plan curricular define género discursivo como una forma de comunicación reconocida como tal por una comunidad de hablantes, más específicamente, hace referencia a clases de textos identificados por el hablante como tales a lo largo de la historia. A su vez, define texto como un fragmento de lengua utilizado para llevar a cabo un acto o acontecimiento comunicativo de tipo discursivo (Instituto Cervantes 2006: 305).

De forma general, los planteamientos que se relacionan con la incorporación del análisis discursivo en el aula de ELE han estado enmarcados en la necesidad de ir más allá de la sistematización de reglas de la lengua y desarrollar, más bien, una competencia discursiva, en primera instancia, oral.

Alcón Soler (2000), siguiendo esta línea de estudio, analiza la comunicación oral en el aula de inglés como lengua extranjera como marco para la enseñanza y la adquisición de la competencia discursiva. La autora, a partir de los resultados, retoma la necesidad de explicitar los mecanismos que caracterizan la interacción oral y concluye que la reflexión metadiscursiva es necesaria para que el estudiante tome conciencia del funcionamiento de la conversación.

Briz, por su parte, destaca la importancia de incluir en el aula de ELE aspectos propios de la pragmática, en tanto esta plantea las estrategias que se siguen al usar la lengua en una situación determinada y "es preciso enseñar también, junto a las categorías y normas gramaticales, las categorías y funciones pragmáticas que rigen la interacción, pues constituyen modos de expresión no solo lingüísticos, sino también socio-culturales" (2002: 13).

Rescatando también la relación entre la pragmática y la enseñanza del español como segunda lengua, Murillo (2004: 259) afirma que las propuestas metodológicas para enseñar segundas lenguas se han centrado, sobre todo, en componentes como el fonético, el morfosintáctico y el léxico, que deben ser descritos y aprendidos, lo cual ha dejado poco espacio para el estudio de la lengua en contextos reales. Para validar la pertinencia de abordar conceptos de pragmática en el aula de ELE, Murillo cita un estudio de Bardovi-Harlig (2001) en el cual se concluye que es necesario enseñar la pragmática en el aula, pues los estudiantes que no pasan por este proceso difieren significativamente de los hablantes nativos en su capacidad 
y desarrollo pragmáticos. Años antes, en 1993, Schmidt había planteado que las funciones pragmáticas y los factores contextuales a menudo no eran tan evidentes en un medio natural aun cuando los aprendices hubieran pasado mucho tiempo inmersos en la cultura meta.

En su investigación, Murillo elabora cuestionarios que plantean situaciones hipotéticas basadas en contextos particulares, con el fin de obtener información sobre el comportamiento verbal de un grupo de estudiantes de nivel avanzado de origen estadounidense. Estos estudiantes aprendían español con el fin de servir como misioneros de una orden religiosa en América Latina. El objetivo básico del estudio consistía en valorar cuáles serían las estrategias de cortesía usadas por estos hablantes en ciertas situaciones comunicativas.

A partir de los datos, el investigador concluye que los estudiantes carecen de entrenamiento en el uso de estrategias pragmáticas en la conversación.

Siguiendo esta misma línea de estudio, Urbina Vargas (2004) propone el análisis del reconocimiento y la percepción de dos fórmulas de saludos informales empleadas en un acto de habla cortés: "un día de estos llegate a mi casa” y "un día de estos hagamos algo". Urbina realiza para ello un cuestionario a 20 hablantes no nativos del español de nivel avanzado, con estudios formales en español como segunda lengua y que hubieran permanecido en Costa Rica al menos tres meses. Para contextualizar las dos fórmulas de cortesía, empleó un diálogo en el cual se estableció una relación informal y sin jerarquías entre los interlocutores.

La autora llega a la conclusión, a partir del contraste entre los resultados obtenidos en hablantes nativos y aprendices del español como segunda lengua, que estos últimos se orientan hacia el elemento proposicional, es decir, hacia el sentido literal de la estructura gramatical, mientras que los nativos tienden a interpretar los enunciados como parte de una situación, puntualmente, como una fórmula convencional de despedida. El estudio corrobora, de esta forma, la necesidad de incorporar en el aula de ELE estrategias pragmáticas que les permitan a los estudiantes comunicarse idóneamente en situaciones comunicativas específicas.

\subsection{La conversación espontánea como material de estudio en el aula de ELE}

Los estudios discursivos se caracterizan por tomar como objeto de análisis datos obtenidos en su entorno "natural" de aparición. Partiendo de esta premisa, hemos elegido el análisis de una conversación espontánea para ejemplificar la aplicación del enfoque discursivo en el aula de ELE, dado que la conversación se caracteriza por ser "la forma primera, primaria y universal de realización de la oralidad; la forma más característica en que las personas se relacionan y llevan a cabo sus actividades cotidianas como seres sociales" (Calsamiglia y Tusón 1999: 32).

Dentro de la tipología discursiva que propone Serrano (2002: 193), esta cabe dentro de lo que denomina tertulia, la cual está determinada por su tono distendido y desordenado, en tanto los turnos de habla se superponen y hay muchas interrupciones y cambios de tema.

Según Kerbrat-Orecchioni (citado en Calsamiglia 1999: 32), este tipo de conversación implica un número relativamente restringido de participantes que no tienen papeles predeterminados y que gozan todos en principio de los mismos derechos y deberes (la interacción es de tipo simétrico e igualitario) Tiene un carácter, a su vez, familiar e improvisado: los temas que se abordan, la duración del intercambio o el orden de los turnos de palabra se determina paso a paso, de forma relativamente libre.

Briz (1998: 42) caracteriza la conversación, frente a otro tipo de discurso hablado, con los siguientes rasgos: 
a) Interlocución en presencia, conversación cara a cara

b) Inmediata, actual (aquí y ahora)

c) Con toma de turno no predeterminada

d) Dinámica, con alternancia de turnos inmediata

e) Cooperativa en relación con el tema de conversación y la intervención del otro.

Podemos decir, entonces, que la conversación espontánea presenta, en general, una planificación sobre la marcha, tiene una finalidad interpersonal (un fin comunicativo socializador) y se manifiesta en un tono informal, no especializado.

Aplicando las premisas de la gramática discursiva, se propone el análisis de una conversación espontánea a partir de un punto específico: el uso discursivo de los pronombres personales tónicos, primera y segunda persona singular.

\subsection{Hacia una propuesta de análisis discursivo de los pronombres personales en el aula de ELE}

En el caso del español, sabemos que la marca morfológica del sujeto la determina la flexión verbal. Ello permite que podamos omitir el pronombre personal correspondiente. Sin embargo, en el discurso podemos determinar que esta opción está reglada por el contexto y no se da de manera casual y completamente arbitraria.

Precisamente, en la conversación se evidencian estrategias discursivas que haremos evidentes.

\subsubsection{La deixis}

Partimos de que todos los enunciados se producen en un contexto particular, el cual influye en su producción y recepción. La deixis se define, precisamente, como "el proceso mediante el cual ciertas unidades lingüísticas remiten directamente a las coordenadas de un acto comunicativo: es decir, al lugar, tiempo y participantes" (Alcaraz Varó y Martínez Linares, 2004: 189). Así, las palabras deícticas refieren a las circunstancias del acto comunicativo y necesitan de ese contexto para alcanzar su sentido pleno en el discurso (Martínez Ruiz, 2000: 243).

La deixis engloba el uso de los pronombres personales, de los demostrativo y de los tiempos verbales, así como de adverbios específicos de tiempo y lugar que señalan la situación del momento de enunciación.

Los pronombres personales pertenecen a la deixis denominada ad oculos, también conocida como deixis exofórica, la cual refiere a una indicación hacia elementos presentes en el contexto del discurso, es decir, que apuntan hacia una realidad extralingüística. Precisamente, los pronombres personales señalan a las personas presentes en la realidad concreta del acto comunicativo.

Martínez Ruiz (2000: 252) ubica a los pronombres personales en la categoría específica de deixis personal. Al respecto, señala que la categoría de persona surge en la lengua a través de dichos pronombres y de las desinencias verbales, indicando las conmutaciones de la enunciación dentro del discurso.

Es de particular interés para nuestro estudio el señalamiento que hace la autora acerca de que la gramática tradicional ha afirmado, durante mucho tiempo, que debido a la transparencia 
de las marcas flexivas, en español no es necesario explicitar el sujeto pronominal. Sin embargo, apunta que en la conversación espontánea su aparición es "frecuente e insistente".

Dado que una de las premisas de la gramática discursiva es indagar en el porqué del uso de determinadas estrategias, nos interesa proponer una serie de posibles causas de este uso a partir de un fragmento de conversación espontánea.

\subsubsection{Los pronombres personales desde una perspectiva discursiva-funcional: expresión y omisión}

Fernández Soriano (1999: 1211) apunta como, a lo largo de toda la tradición gramatical, el pronombre ha sido una de las categorías que ha suscitado más polémica en cuanto a su definición y adscripción a una determinada clase. La autora ejemplifica esta afirmación con diferentes posturas: Bello (1847) no le confiere independencia y lo analiza en conjunto con el artículo. La RAE, por su parte, agrupa al pronombre junto con el nombre.

En nuestro análisis partiremos de que el pronombre pertenece a la clase del sustantivo. Una justificación de esta afirmación es el hecho de que el pronombre personal desempeña las mismas funciones sintácticas que el sustantivo. Sin embargo, contrario a los sustantivos, carece de contenido semántico inherente, dado que adquiere significado dependiendo de las circunstancias del discurso. Dentro de los pronombres, serán objeto de nuestro análisis los pronombres de la serie tónica en función de sujeto.

La gramática tradicional ha afirmado por mucho tiempo que la aparición de un pronombre explícito en posición de sujeto es superflua, en tanto sus condiciones de aparición se relacionan con factores de redundancia, énfasis y ambigüedad, los cuales se explican por razones estilísticas más que gramaticales. Esta postura confirma, una vez más, el criterio de separar y aislar el componente gramatical de otros componentes de la lengua, como el pragmático-discursivo.

Desde una perspectiva funcional, sin embargo, no hay alternancia indiscriminada entre el uso y la omisión de los pronombres sujeto. En cuanto a su omisión, tenemos ejemplos como los siguientes, en donde el pronombre no es redundante, sino más bien casi inaceptable discursivamente:

"Yo fui al banco y después yo fui a la panadería, pero aunque yo me apuré yo llegué muy tarde a la casa"

En este caso, no podría hablarse de que el uso de los pronombres explícitos sea completamente opcional. Interesa, más bien, investigar los factores que determinan su posibilidad de aparición.

\subsubsection{El material sujeto a análisis}

\subsubsection{Descripción}

La conversación cuenta con la participación de dos hablantes nativos del español y tiene lugar en una casa de habitación. Ambos participantes tienen edades similares, uno de ellos 35 y el otro 37 años. Los dos hablantes son costarricenses y se desempeñan como técnicos de telecomunicaciones. El tema de la conversación es la realización de reuniones por 
parte de la pastoral de la iglesia de la comunidad. La grabación fue secreta y posteriormente se les solicitó a los participantes el permiso respectivo para utilizarla con fines académicos. Para propósitos de este estudio, se extraen dos minutos de una conversación de 5 minutos, aproximadamente.

\subsubsection{Transcripción}

Para la transcripción, se siguen los lineamientos de Calsamiglia y Tusón (1999:360):

a) Uso de ortografía estándar.

b) No se utilizan de mayúsculas (salvo en nombres propios) ni signos de puntuación.

c) Las cifras a la izquierda del texto indican el número de la toma de palabra.

d) Las iniciales en mayúscula identifican a cada participante: H1: hablante 1, H2: hablante 2.

e) Los símbolos / // // indican pausas de diferente duración.

f) [ ] Alrededor de un fragmento indica que esa secuencia se solapa con las palabras de otro participante, que se reproducen también entre corchetes.

g) $\quad==\mathrm{Al}$ comienzo de una toma de palabra indica que no existe pausa entre esa toma

y la anterior. Cualquier aspecto relevante en cuanto a elementos no verbales se destaca entre corchetes.

Con el fin de analizar las diferentes apariciones de los pronombres personales, estas se han numerado, se encuentran en negrita y subrayadas, y se sigue este formato al momento de su análisis.

Dado que en algunos casos la estrategia que implica la aparición de determinados pronombres es la misma, hemos decidido colocar primero el nombre de la estrategia discursiva utilizada, seguido por el (los) caso (s) que la ejemplifica (n) a lo largo de la conversación y, por último, la explicación de dicha estrategia.

1. H1: $\quad$ Según el señor son diez reuniones a las que 1-vos tenés que ir.

2. H2: = = ¿diez? / ¿con qué tiempo? / 2-yo no sé cómo hace la gente / cómo hace la gente para sacar tiempo para eso [de veras que hay que estar de vago]

3. H1: [diay saca tiempo / tiempo hay]

4. H2: ¿ ¿Y va uno y qué?

5. H1: Va y vas a ver que te va mejor en todo // 3-yo creo que uno cambia / cambia / como más paciencia [ uno tiene / sí / más paciencia ]

6. H2: [4-yo sabía] al final es aguantar todo

7. H1: $\quad$ ah / [Risas] no no / 5-no entendés vos / 6- voy a explicarle mejor a tu doña yo

8. H2: [Risas] Explicame, explicame a ver

9. H1: $\quad$ 7-Yo explicarte / te explico / pero $\underline{8-\text { no creés en nada vos }}$

10. H2: = = no / pero 9- yo creo que sí hace falta creer / como tener fe / como fe en algo 
11. H1: = = sí sí / pero hay que agarrarlo en serio / uno se compromete y va a las reuniones // hay retiros también y así va metiendo a la familia

12. $\mathrm{H} 2$ : $\quad$ sí / pero 10 - yo pienso que una cosa es creer en eso y otra engañarse uno mismo // mirá la gente que pasa yendo a la iglesia y es peor que uno

13. H1: bueno, gente así / gente así siempre hay

14. H2: = = alcanzame un vaso si me hacés el favor

\subsubsection{Análisis del uso de los pronombres personales}

Como se indicó antes, se coloca primero el nombre de la estrategia discursiva utilizada, seguido por el (los) caso (s) que la ejemplifica (n) y, por último, la explicación de dicha estrategia.

A- Uso del pronombre para indicar referencia genérica: 1-vos tenés que ir:

En este caso, podríamos hablar de la estrategia del uso del pronombre personal como referencia genérica. Así, a través de la generalización de la experiencia, el hablante involucra al oyente y señala que esta experiencia es compartida por otros. Es claro que el requisito de tener que ir a diez reuniones aplica a toda la gente interesada en ir, y no solo a la segunda persona, representada por vos.

B- Toma de posición personal en el enunciado: 2- yo no sé cómo hace la gente, 3-yo creo que uno cambia, 4- yo sabía, 9- yo creo que sí hace falta creer, 10- yo pienso que una cosa es creer en eso

Encontramos en estos ejemplos una toma de posición personal en el enunciado, característica de verbos de conocimiento y opinión, es el caso de saber, creer o pensar. La aparición del pronominal sujeto se podría explicar, en estos casos, por la clase semántica a la que pertenece el verbo: campo del conocimiento o percepción intelectual.

C- $\quad$ Énfasis en el cierre del enunciado: 5- no entendés vos, 6- vov a explicarle mejor a tu doña yo y 8-

La estrategia de colocar el pronombre personal pospuesto al verbo, lo enfatiza al cierre del enunciado y recupera la atención en él.

D- $\quad$ Marca de sujeto ante forma no finita: 7- yo explicarte / te explico:

La forma no flexionada favorece la aparición de la señal morfológica de sujeto mediante el pronombre, al tiempo que se extiende y enfatiza el contenido léxico del verbo.

Como vemos, el partir del análisis de una conversación permite identificar y plantear posibles estrategias discursivas. Limitarse al análisis gramatical en el nivel de la oración solamente, perpetuaría la visión de que el uso o no del pronombre es puramente opcional y, por ende, dejaría de lado las implicaciones que tiene en la conversación.

De acuerdo con Serrano (2002: 81), podemos confirmar cómo "la capacidad formal de expresar la co-indización de la persona gramatical con la flexión verbal adquiere, ya en el uso, la función discursiva de representar intenciones y vinculaciones del hablante con el contenido cognitivo al que refiere". 
Esta posición, que es la que se sigue en nuestro estudio, descarta la visión tradicional que plantea que los pronombres son formas opcionales concordantes y subsidiarias, puesto que se puede evidenciar, en una muestra de habla real, que más bien está motivada por las funciones que quieran expresar los hablantes en un espacio físico concreto y en un tiempo determinado. El análisis de estas funciones, está de más decir, les permitiría a los estudiantes de ELE lograr una visión más integral y completa de la organización que presentan los textos orales.

\section{Conclusiones}

En la mayoría de los casos, esperamos de los estudiantes de ELE un desenvolvimiento apropiado en situaciones de conversación concretas, pero obviamos el estudio de aspectos de uso de la lengua que determinan, precisamente, la naturalidad de tales intercambios comunicativos.

El estudio de estrategias discursivas en conversaciones espontáneas pone de manifiesto que la perspectiva de la gramática oracional no es suficiente para analizar la regularidad de los patrones de comunicación oral. La gramática discursiva, en cambio, nos permite explicar cómo funciona una gramática a partir del estudio de estructuras que alternan, o bien, parecen alternar, para luego probar su similitud sintáctica y pragmática, su aparición en contextos, su uso dentro de determinada comunidad de habla y su relación con factores sociales específicos.

Si en realidad incorporamos en el aula de ELE una noción discursiva de la gramática, necesariamente deberemos entonces tomar en cuenta las categorías que pertenecen a esta perspectiva: el hablante y el oyente, el acto que realizan al producir un enunciado o al recibirlo y la finalidad que este tiene, el sistema lingüístico empleado, las actitudes de los interlocutores y los sistemas de normas y obligaciones marcados socialmente.

Un análisis de tipo discursivo en el aula nos llevará a identificar tipos de discurso, así como rasgos situacionales y lingüísticos que denotan determinada modalidad.

Si continuamos basándonos en la perspectiva de la gramática oracional, limitándonos al estudio de patrones estructurales basados en oraciones construidas a propósito y no pasamos al plano discursivo, estaremos promoviendo en el aula de ELE reglas que están lejos de manifestarse de forma constante y rígida en intercambios comunicativos reales, tal sería, por ejemplo, la regla de que la aparición de un pronombre explícito en posición de sujeto es superflua y obedece a razones de énfasis o redundancia. Como se comprobó, esta dista de ser verdadera en la muestra de conversación espontánea que tomamos en cuenta para nuestra propuesta de análisis.

Concluimos retomando la posición de De Beaugrande y Wolfgang, con respecto a la importancia del análisis a nivel discursivo:

\footnotetext{
Las palabras y oraciones que aparecen literalmente en un texto son indicaciones interesantes que ha de tener muy en cuenta el analista, pero no reproducen la totalidad de lo que se está comunicando, por lo que si en nuestro análisis nos limitásemos a ellas nunca podríamos ofrecer una descripción completa de cómo funciona un texto. Y precisamente el problema más apremiante que ha de resolverse es cómo funcionan los textos en la interacción comunicativa. (1997: 35)
}

\section{Notas}

1. Larsen-Freeman y Long (1994: 17) apuntan a una distinción entre el término lengua extranjera y segunda lengua, la cual se basa en el contexto de aprendizaje. Así, la segunda lengua se adquiere en un entorno donde funciona como lengua nativa, mientras que la lengua extranjera se adquiere fuera de este 
entorno, es decir, donde no es lengua nativa. Dado que esta distinción se utiliza para profundizar en la diferencia entre adquirir y aprender una lengua, y algunas veces los términos se utilizan indistintamente, decidimos emplear el término español como lengua extranjera (siglas ELE) por resultar más abarcador, de acuerdo con los objetivos de este trabajo.

2. La distinción entre aprender y adquirir una lengua, también tratada a profundidad por Larsen-Freeman y Long (1994), no será tampoco determinante en nuestro estudio, en tanto elegiremos el término aprender por estar asociado con la enseñanza formal de una lengua, en un aula y siguiendo un programa específico.

3. La gramaticalización se define como el cambio en la competencia procesual del hablante, por medio del cual una parte del conocimiento lingüístico empieza a procesarse de manera automática (Butler, Christopher 1999:23).

4. Esta distinción se corresponde con otras dicotomías funcionales, tales como la ideacional / interpersonal de Halliday y la descriptiva / social expresiva de Lyons.

\section{Bibliografía}

Alarcos Llorach, Emilio. 2000. Gramática de la lengua española. Madrid: Espasa- Calpe.

Alcaraz Varó, Enrique y María Antonia Martínez Linares. 2004. Diccionario de Lingüística Moderna. Barcelona: Ariel, S.A.

Alcón Soler, Eva. 2000. "Desarrollo de la competencia discursiva oral en el aula de lenguas extranjeras: perspectivas metodológicas y de investigación”. En: Muñoz, Carmen (eds.), 259-272.

Bardovi-Harlig. 2001. "Evaluating the empirical evidence: Grounds for instruction in pragmatics?”. En: Rose, K. y Gabriele Kasper (eds.), 13-32.

Bello, Andrés. 1847. Gramática de la lengua castellana destinada al uso de los americanos. (20 Ed). Tenerife: Aula Cultural de España.

Bosque, Ignacio y Violeta Demonte (dir.) 2000. Gramática descriptiva de la lengua española. Vol.1. Madrid: Espasa Calpe.

Briz, Antonio. 1998. El español coloquial en la conversación. Esbozo de pragmagramática. Barcelona: Ariel, S.A.

2000. ¿Cómo se comenta un texto coloquial? Barcelona: Ariel Practicum.

2002. El español coloquial en la clase de E/LE. Un recorrido a través de los textos. Madrid: Sociedad General Española de Librería, S.A.

Brown, Gillian y George Yule. 1993. Análisis del discurso. Madrid: Visor Libros. 
Calsamiglia Blancafort, Helena y Ampara Tusón Valls. 1999. Las cosas del decir. Manual de análisis del discurso. Barcelona: Ariel, S.A.

Celce-Murcia Marianne, Olshtain, Elite. 2000. Discourse and context in language teaching. Cambridge: Cambridge University Press.

Crystal, David. 1997. The Cambridge encyclopedia of Language. United Kingdom: Cambridge University Press.

Cumming, Susanna y Tsuyoshi Ono. 2000. “El discurso y la gramática”. En: Van Dijk (comp.), 13-32.

De Beaugrande, Robert Alain y Wolfgag Ulrich Dressler. 1997. Introducción a la lingüística del texto. Barcelona: Ariel, S.A.

Escandel Vidal, M.V. 1996. Introducción a la pragmática. Barcelona: Ariel Lingüística.

Fernández, Olga. 1999. "El pronombre personal. Formas y distribuciones. Pronombres átonos y tónicos”. En: Bosque y Demonte (dir.), 1211-1269.

Givon, T. 1995. Syntax: a Typological Functional Introduction, Vol. I. Amsterdam and Philadelphia: John Benjamins.

Halliday, M.A.K. 1961. Categories of the theory of grammar. Londres: Continuum.

1973. Explorations in the Funtions of Language. Londres: Edward Arnold.

Instituto Cervantes. 2006. Plan curricular del Instituto Cervantes. Niveles de referencia para el español. Madrid: Editorial Biblioteca Nueva, S.L.

Kasper, Gabriele y Blum-Kulka, S. 1993. Interlanguage Pragmatics. Oxford: Oxford University Press.

Kotschi, Thomas, Wulf Oesterreicher y Klaus Zimmermann (eds.). 1996. El español hablado y la cultura oral en España e Hispanoamérica. Madrid: Vervuert.

Larsen-Freeman, Diane y Michael H. Long. 1994. Introducción al estudio de la adquisición de segundas lenguas. Madrid: Gredos.

Martínez Ruiz, Raquel. 2000. “La deixis”. En: Briz, Antonio (comp.), 243-262.

Martínez, María Cristina. 2001. Análisis del discurso y práctica pedagógica. Argentina: Homo Sapiens Ediciones. 
Muñoz, Carmen (Ed). 2000. Segundas Lenguas. Adquisición en el aula. Barcelona: Editorial Ariel, S.A.

Murillo Medrano, Jorge. 2004. "La pragmática y la enseñanza del español como segunda lengua”. Revista de Educación de la Universidad de Costa Rica. 28 (2): 255-267.

Rose, K. y Gabriele Kasper. 2001. Pragmatics in Language Teaching. Nueva York: Cambridge University Press.

Sánchez Avendaño, Carlos. 2006. "La forma del grupo nominal en español hablado: un caso de gramática del discurso frente a gramática de la palabra". Revista de Filología y Lingüística de la Universidad de Costa Rica. 32 (2): 261-289.

Sánchez Perez, Aquilino. 1997. Los métodos en la enseñanza de idiomas. Madrid: Sociedad General Española de Librería S.A.

Schmidt, R. 1993. “Consciousness, learning and interlanguage pragmatics”. En: Kasper, G y Blum-Kulka, S. (eds.) 15-44.

Schriffin, Deborah. 1994. Approaches to discourse. Cambridge: Blacwell.

Serrano, María José. 2002. Aproximación a la gramática del discurso del español. Munich: LINCOM Studies in Romance Linguistics.

Shrum, J. y Glisan, E. 1994. Teacher's Handbook. Contextualized Language Instruction. Boston: Heinle and Heinle Publishers.

Urbina Vargas, Soledad. 2004. “Análisis pragmático de dos expresiones de cortesía del español de Costa Rica y su adquisición por parte de estudiantes de español como segunda lengua". Revista de Filología y Lingüística de la Universidad de Costa Rica. 30 (2): 237-245.

Van Dijk, Teun (comp.). 2000. El discurso como estructura y proceso. Barcelona: Gedisa.

Sánchez, Aquilino. 1997. Los métodos en la enseñanza de idiomas. Madrid: Sociedad General Española de Librería, S.A. 